\title{
Article
}

\section{A Note on a Fourier Sine Transform}

\author{
Robert Reynolds *(1) and Allan Stauffer (1)
}

Department of Mathematics and Statistics, York University, Toronto, ON M3J 1P3, Canada; stauffer@yorku.ca

* Correspondence: milver@my.yorku.ca

\begin{abstract}
This is a compilation of definite integrals of the product of the hyperbolic cosecant function and polynomial raised to a general power. In this work, we used our contour integral method to derive a Fourier sine transform in terms of the Lerch function. Almost all Lerch functions have an asymmetrical zero-distribution. A summary table of the results are produced for easy reading. A vast majority of the results are new.
\end{abstract}

Keywords: entries in Gradshteyn and Ryzhik; definite integral; contour integral

MSC: Primary 30E20; 33-01; 33-03; 33-04; 33-33B; 33E20

\section{Significance Statement}

Definite integrals involving hyperbolic functions and a polynomial have been studied by Ramanujan [1], and play a role in the theory of heavy-ion elastic collisions [2], study the dislocation or nodal lines of 3D Berry's random waves mod [3], study the heat losses and temperature in the ground under a building with and without ground water flow [4], and used in books such Advanced Calculus: Of Real-valued Functions of a Real Variable and Vector-valued Functions of a Vector Variable [5].

Based upon current literature there is vast usage of such integral formulae and hence

Citation: Reynolds, R.; Stauffer, A. A Note on a Fourier Sine Transform. Symmetry 2021, 13, 1828. https:// doi.org/10.3390/sym13101828

Academic Editor: Dongfang Li

Received: 11 September 2021

Accepted: 26 September 2021

Published: 30 September 2021

Publisher's Note: MDPI stays neutral with regard to jurisdictional claims in published maps and institutional affiliations. derive generalized forms of definite integrals involving the hyperbolic cosecant function and a polynomial raised to a power and express it in terms of the the Lerch function, hence the aim of this current work (see [6,7]) and reference therein. This integral formula is then used to derive a table of definite integrals which aims at expanding current textbooks featuring such integrals. This resource will be beneficial to researchers requiring such formulae for their work.

\section{Introduction}

We apply the simultaneous contour integral method [8] to an integral Equation (3.911.3) in [9] and use it to derive closed forms in the book of Brychkov et al. [10] along with new integral formulae. In this paper, we derive the definite integral given by

$$
\int_{0}^{\infty} \operatorname{csch}\left(\frac{x}{2}\right)\left(e^{m x}(\log (a)-i x)^{k}-e^{-m x}(\log (a)+i x)^{k}\right) d x
$$

and used it to achieve several objectives. We derive formula for the Mellin transform, Fourier cosine, and sine transform. We offer formal derivations for various definite integrals in [9]. We also provide a Table 1 of transforms that is not already included in current literature to summarize our results. The Mellin transform is utilized in practically every field of research and engineering, including statistics and geophysical data processing, to mention a few. The constants $a, k, c$, and $m$ in Equation (1) are general complex numbers 
subject to the conditions stated. The derivations follow the method used by us in [8]. The generalized Cauchy's integral formula is given by

$$
\frac{y^{k}}{\Gamma(k+1)}=\frac{1}{2 \pi i} \int_{C} \frac{e^{w y}}{w^{k+1}} d w
$$

where $C$ is in general an open contour in the complex plane where the bilinear concomitant [8] has the same value at the end points of the contour and the gamma function is defined in Equation (5.2.1) in [11].

Table 1. Table of Integrals.

\begin{tabular}{|c|c|}
\hline$f(x)$ & $\int_{0}^{\infty} f(x) d x$ \\
\hline $\operatorname{csch}\left(\frac{x}{2}\right) \sin (m x)$ & $\pi \tanh (\pi m)$ \\
\hline$\frac{\operatorname{csch}\left(\frac{x}{2}\right)(\log (a) \sinh (m x)+i x \cosh (m x))}{\log ^{2}(a)+x^{2}}$ & $\frac{i\left(\pi-e^{2 i \pi m} \log (a) \Phi\left(-e^{2 i m \pi}, 1, \frac{\log (a)}{2 \pi}+1\right)\right)}{\log (a)}$ \\
\hline$x^{s-1} \operatorname{csch}\left(\frac{x}{2}\right) \sinh (m x)$ & $i 2^{s-1} \pi^{s} \csc \left(\frac{\pi s}{2}\right)\left(\operatorname{Li}_{1-s}\left(-e^{2 i m \pi}\right)-\operatorname{Li}_{1-s}\left(-e^{-2 i m \pi}\right)\right)$ \\
\hline$x^{s-1} \sinh (a x) \operatorname{csch}(b x)$ & $\frac{1}{2} i \pi^{s} b^{-s} \csc \left(\frac{\pi s}{2}\right)\left(\operatorname{Li}_{1-s}\left(-e^{\frac{i a \pi}{b}}\right)-\operatorname{Li}_{1-s}\left(-e^{-\frac{i a \pi}{b}}\right)\right)$ \\
\hline$x^{s-1} \cosh (a x) \operatorname{csch}(b x)$ & $\frac{1}{2} \pi^{s} b^{-s} \sec \left(\frac{\pi s}{2}\right)\left(\operatorname{Li}_{1-s}\left(-e^{-\frac{i a \pi}{b}}\right)+\operatorname{Li}_{1-s}\left(-e^{\frac{i a \pi}{b}}\right)\right)$ \\
\hline$\frac{\operatorname{csch}(b x) \sinh (m x)}{a^{2}+x^{2}}$ & $\frac{i e^{-\frac{i \pi m}{b}}\left(\Phi\left(-e^{-\frac{i m \pi}{b}}, 1, \frac{a b}{\pi}+1\right)-e^{\frac{2 i \pi m}{b}} \Phi\left(-e^{\frac{i m \pi}{b}}, 1, \frac{a b}{\pi}+1\right)\right)}{32 a b^{3}}$ \\
\hline$\frac{x \operatorname{csch}(\pi x) \cos (m x)}{a^{2}+x^{2}}$ & $-\frac{a e^{m} \Phi\left(-e^{m}, 1, a+1\right)+a e^{-m} \Phi(\sinh (m)-\cosh (m), 1, a+1)-1}{32 \pi^{3} a}$ \\
\hline$\frac{(1-\cosh (p x)) \operatorname{csch}(q x)}{x}$ & $\log \left(\cos \left(\frac{\pi p}{2 q}\right)\right)$ \\
\hline$x^{2}(\sinh (p x)-1) \operatorname{csch}(q x)$ & $\frac{\pi^{3} \tan \left(\frac{\pi p}{2 q}\right) \sec ^{2}\left(\frac{\pi p}{2 q}\right)-14 \zeta(3)}{4 q^{3}}$ \\
\hline $\operatorname{csch}(b x)(\sin (c x)-\sin (a x))$ & $\frac{\pi\left(\tanh \left(\frac{\pi c}{2 b}\right)-\tanh \left(\frac{\pi a}{2 b}\right)\right)}{2 b}$ \\
\hline$\frac{\operatorname{csch}(b x)(\cos (c x)-\cos (a x))}{x}$ & $\log \left(\cosh \left(\frac{\pi a}{2 b}\right) \operatorname{sech}\left(\frac{\pi c}{2 b}\right)\right)$ \\
\hline$x \operatorname{csch}(b x)(\sinh (c x)-\cosh (a x))$ & $-\frac{\pi}{b^{2}}\left(\frac{\pi}{2 \cos \left(\frac{\pi a}{b}\right)+2}+i\left(L i_{-1}^{\prime}\left(-e^{-\frac{i \pi c}{b}}\right)-L i_{-1}^{\prime}\left(-e^{\frac{i \pi c}{b}}\right)\right)\right)$ \\
\hline$\frac{\operatorname{csch}\left(\frac{x}{2}\right) \sinh (m x) \sinh (p x)}{x}$ & $\frac{1}{2} \log (\cos (\pi(m-p)) \sec (\pi(m+p)))$ \\
\hline $\operatorname{csch}\left(\frac{x}{2}\right) \cosh (m x) \sinh (p x)$ & $\frac{1}{2} \pi(\tan (\pi(m+p))-\tan (\pi(m-p)))$ \\
\hline $\operatorname{csch}(\pi x) \cos (n x) \sinh (p x)$ & $\frac{\sin (p)}{2(\cosh (n)+\cos (p))}$ \\
\hline
\end{tabular}

\section{Definite Integral of the Contour Integral}

Using the method in [8] involving Cauchy's integral Equation (2), we replace $y$ by $\log (a)+i x$ and multiply both sides by $e^{m x i}$. Then, we derive a second equation by replacing $x$ by $-x$ and adding, followed by multiplying both sides by $\frac{i e^{x / 2}}{2\left(e^{x}-1\right)}$ to get 


$$
\begin{aligned}
& \frac{1}{\Gamma(k+1)} \int_{0}^{\infty} \operatorname{csch}\left(\frac{x}{2}\right)\left(e^{m x}(\log (a)-i x)^{k}-e^{-m x}(\log (a)+i x)^{k}\right) d x \\
& =\frac{1}{2 \pi i} \int_{0}^{\infty} \int_{C} \frac{e^{x / 2} a^{w} w^{-k-1} \sin (x(m+w))}{e^{x}-1} d w d x \\
& =\frac{1}{2 \pi i} \int_{C} \int_{0}^{\infty} \frac{e^{x / 2} a^{w} w^{-k-1} \sin (x(m+w))}{e^{x}-1} d x d w \\
& =\frac{1}{2 \pi i} \int_{C} \frac{1}{2} \pi a^{w} w^{-k-1} \tanh (\pi(m+w)) d w
\end{aligned}
$$

from Equation (3.911.3) in [9] where $\operatorname{Re}(m+w)>0$. We may use Fubini's theorem to change the order of integration between $w+m$ and $x$ because the integrand has a bounded measure over the space $\mathbb{C} \times[0, \infty)$, where $\mathbb{C}$ is the set of all non-zero complex numbers.

\section{The Lerch Function}

The Lerch function $\Phi(z, s, v)$, see Equation (1.11.3) in [12] is a generalization of the Hurwitz zeta and Polylogarithm functions is given by:

$$
\Phi(z, s, v)=\sum_{n=0}^{\infty}(v+n)^{-s} z^{n}
$$

where $|z|<1, v s . \neq 0,-1,-2,-3, . .$, and is continued analytically by its integral representation given by

$$
\Phi(z, s, v)=\frac{1}{\Gamma(s)} \int_{0}^{\infty} \frac{t^{s-1} e^{-(v-1) t}}{e^{t}-z} d t
$$

where $\operatorname{Re}(v)>0$, and either $|z| \leq 1, z \neq 1, \operatorname{Re}(s)>0$, or $z=1, \operatorname{Re}(s)>1$.

\section{Infinite Sum of the Contour Integral}

Again, we use the method in [8]. Using Equation (2) replace $y$ by $\log (a)+2 \pi(y+1)$ and multiply both sides by $(-1)^{y} e^{2 \pi m(y+1)}$. Next, we take the infinite sum over $y \in[0, \infty)$ and simplify in terms of the Lerch function to get

$$
\begin{aligned}
& \frac{2^{k} \pi^{k+1} e^{2 \pi m} \Phi\left(-e^{2 m \pi},-k, \frac{\log (a)}{2 \pi}+1\right)}{\Gamma(k+1)} \\
& =\frac{1}{2 \pi i} \sum_{y=0}^{\infty} \int_{C} \pi(-1)^{y} a^{w} w^{-k-1} e^{2 \pi(y+1)(m+w)} d w \\
& =\frac{1}{2 \pi i} \int_{C} \sum_{y=0}^{\infty} \pi(-1)^{y} a^{w} w^{-k-1} e^{2 \pi(y+1)(m+w)} d w \\
& =\frac{1}{2 \pi i} \int_{C} \frac{1}{2} \pi a^{w} w^{-k-1} \tanh (\pi(m+w))+\frac{1}{2} \pi a^{w} w^{-k-1} d w
\end{aligned}
$$

from Equation (1.232.1) in [9] where $\tanh (i x)=i \tan (x)$ from (4.5.9) in [13] and $\operatorname{Im}(m+$ $w)>0$ for the convergence of the sum.

\subsection{Additional Contour}

Next we derive the additional contour by using Equation (2) replacing y by $\log (a)$ and multiplying by $\frac{\pi}{2}$ to get

$$
\frac{\pi \log ^{k}(a)}{2 \Gamma(k+1)}=\frac{1}{2 \pi i} \int_{C} \frac{1}{2} \pi a^{w} w^{-k-1} d w
$$




\subsection{Definite Integral in Terms of the Lerch Function}

Theorem 1. For all $k, a, m \in \mathbb{C}$,

$$
\begin{aligned}
& \int_{0}^{\infty} \operatorname{csch}\left(\frac{x}{2}\right)\left(e^{m x}(\log (a)-i x)^{k}-e^{-m x}(\log (a)+i x)^{k}\right) d x \\
& =2 i \pi\left(\log ^{k}(a)-2^{k+1} \pi^{k} e^{2 i \pi m} \Phi\left(-e^{2 i m \pi},-k, \frac{\log (a)}{2 \pi}+1\right)\right)
\end{aligned}
$$

Proof. Since the right-hand side of Equation (3) is equal to the sum of (6) and (7) we can equate the left-hand sides to yield the stated result.

\section{Main Results}

In the proceeding section we will evaluate Equation (8) in terms of special functions namely; polylogarithm function $L i_{n}(z)$ see Section (25:12) in [11], Riemann zeta function $\zeta(s)$ see Section (25.2) in [11], and Hurwitz zeta function $\zeta(s, a)$ see Section (25.11) [11].

\subsection{Derivation of Entry (3.911.3) in Gradshteyn and Ryzhik}

Proposition 1.

$$
\int_{0}^{\infty} \operatorname{csch}\left(\frac{x}{2}\right) \sin (m x) d x=\pi \tanh (\pi m)
$$

Proof. Use Equation (8) and set $k=0$ and simplify using entry (2) in Table below (64:12:7) in [14] where $\Phi(x, 0, u)=\frac{1}{1-x}$.

\section{Proposition 2.}

$$
\begin{aligned}
& \int_{0}^{\infty} \frac{\operatorname{csch}\left(\frac{x}{2}\right)(\log (a) \sinh (m x)+i x \cosh (m x))}{\log ^{2}(a)+x^{2}} d x \\
& =\frac{i\left(\pi-e^{2 i \pi m} \log (a) \Phi\left(-e^{2 i m \pi}, 1, \frac{\log (a)}{2 \pi}+1\right)\right)}{\log (a)}
\end{aligned}
$$

Proof. Use Equation (8) and set $k=-1$ and simplify using entry (1) in Table below (64:12:7) in [14].

\subsection{Derivation of Entry 2.3.13 in Brychkov}

Proposition 3. For $\operatorname{Re}(b)>|\operatorname{Re}(a)|, \operatorname{Re}(s)>0$,

$$
\begin{aligned}
& \int_{0}^{\infty} x^{s-1} \sinh (a x) \operatorname{csch}(b x) d x \\
& =\frac{1}{2} i \pi^{s} b^{-s} \csc \left(\frac{\pi s}{2}\right)\left(L i_{1-s}\left(-e^{\frac{i a \pi}{b}}\right)-L i_{1-s}\left(-e^{-\frac{i a \pi}{b}}\right)\right)
\end{aligned}
$$

Proof. Use Equation (8) and form a second equation by replacing $m \rightarrow-m$ and take their difference to get

$$
\begin{array}{r}
\int_{0}^{\infty} \operatorname{csch}\left(\frac{x}{2}\right) \sinh (m x)\left((\log (a)-i x)^{k}+(\log (a)+i x)^{k}\right) d x \\
=-i(2 \pi)^{k+1} e^{-2 i \pi m}\left(e^{4 i \pi m} \Phi\left(-e^{2 i m \pi},-k, \frac{\log (a)}{2 \pi}+1\right)\right. \\
\left.-\Phi\left(-e^{-2 i m \pi},-k, \frac{\log (a)}{2 \pi}+1\right)\right)
\end{array}
$$

Next we set $a=1, k=s-1$ and simplify using entry (4) in Table below (64:12:7) in [14] to get 


$$
\begin{aligned}
& \int_{0}^{\infty} x^{s-1} \operatorname{csch}\left(\frac{x}{2}\right) \sinh (m x) d x=i 2^{s-1} \pi^{s} \csc \left(\frac{\pi s}{2}\right) \\
& \left(\operatorname{Li}_{1-s}\left(-e^{2 i m \pi}\right)-\operatorname{Li}_{1-s}\left(-e^{-2 i m \pi}\right)\right)
\end{aligned}
$$

Next replace $x \rightarrow a x, m \rightarrow m /(2 a), a \rightarrow 2 a, b \rightarrow a$ and $a \rightarrow b$ and simplify to get the stated result.

\subsection{Derivation of Entry 2.3.14 in Brychkov}

Proposition 4. For $\operatorname{Re}(b)>|\operatorname{Re}(a)|, \operatorname{Re}(s)>1$,

$$
\begin{aligned}
& \int_{0}^{\infty} x^{s-1} \cosh (a x) \operatorname{csch}(b x) d x \\
& =\frac{1}{2} \pi^{s} b^{-s} \sec \left(\frac{\pi s}{2}\right)\left(L i_{1-s}\left(-e^{-\frac{i a \pi}{b}}\right)+L i_{1-s}\left(-e^{\frac{i a \pi}{b}}\right)\right)
\end{aligned}
$$

Proof. Use Equation (11) and take the first partial derivative with respect to $a$ and replace $s \rightarrow s-1$ and simplify.

Proposition 5. For $\operatorname{Re}(b)>|\operatorname{Re}(m)|, \operatorname{Re}(a)>0$,

$$
\begin{aligned}
& \int_{0}^{\infty} \frac{\operatorname{csch}(b x) \sinh (m x)}{a^{2}+x^{2}} d x \\
& =\frac{i}{2 a} e^{-\frac{i \pi m}{b}}\left(\Phi\left(-e^{-\frac{i m \pi}{b}}, 1, \frac{a b}{\pi}+1\right)-e^{\frac{2 i \pi m}{b}} \Phi\left(-e^{\frac{i m \pi}{b}}, 1, \frac{a b}{\pi}+1\right)\right)
\end{aligned}
$$

Proof. Use Equation (8) and set $k=-1$ and replace $a \rightarrow e^{a}$ and simplify using Equation (9.559) in [9]. Next replace $x \rightarrow b x, m \rightarrow m /(2 b)$ and $b \rightarrow 2 b$ then $a \rightarrow 2 a b$ and simplify. Note by simple parameter substitution, using Equation (15) we can derive entries (3.525.7), (3.525.8), (3.525.1), (3.525.2), (3.525.3) and (3.525.4) in [9].

Lemma 1. For $\operatorname{Re}(m)<\pi, \operatorname{Re}(a)>0$,

$$
\int_{0}^{\infty} \frac{\operatorname{csch}(\pi x) \sin (m x)}{a^{2}+x^{2}} d x=\frac{e^{m}\left(\Phi\left(-e^{m}, 1, a+1\right)-e^{-2 m} \Phi\left(-e^{-m}, 1, a+1\right)\right)}{32 \pi^{3} a}
$$

Proof. Use Equation (15) and set $m=m i, b=\pi$ and simplify.

Lemma 2. For $\operatorname{Re}(m)<\pi, \operatorname{Re}(a)>0$,

$$
\begin{aligned}
& \int_{0}^{\infty} \frac{x \operatorname{csch}(\pi x) \cos (m x)}{a^{2}+x^{2}} d x \\
& =-\frac{a e^{m} \Phi\left(-e^{m}, 1, a+1\right)+a e^{-m} \Phi(\sinh (m)-\cosh (m), 1, a+1)-1}{32 \pi^{3} a}
\end{aligned}
$$

Proof. Use Equation (16) and take the first partial derivative with respect to $m$ and simplify.

Proposition 6. For $\operatorname{Re}(m)<1 / 2, \operatorname{Re}(a)>0$,

$$
\begin{aligned}
& \int_{0}^{\infty} \operatorname{csch}\left(\frac{x}{2}\right) \log \left(a^{2}+x^{2}\right) \sinh (m x) d x \\
& =\frac{2 i \pi}{e^{2 i \pi m}+e^{4 i \pi m}}\left(( 1 + e ^ { 2 i \pi m } ) \left(e^{4 i \pi m} \Phi^{\prime}\left(-e^{2 i \pi m}, 0, \frac{a}{2 \pi}+1\right)\right.\right. \\
& \left.\left.-\Phi^{\prime}\left(-e^{-2 i \pi m}, 0, \frac{a}{2 \pi}+1\right)\right)-e^{2 i \pi m}\left(-1+e^{2 i \pi m}\right) \log (2 \pi)\right)
\end{aligned}
$$


Proof. Use Equation (8) and take the first partial derivative with respect to $k$ and set $k=0$ and $a \rightarrow e^{a}$ and simplify.

Proposition 7.

$$
\begin{aligned}
& \int_{0}^{\infty} x^{s-1} \operatorname{csch}(b x)(\cosh (c x)-\cosh (a x)) d x=-\frac{1}{2} \pi^{s} b^{-s} \sec \left(\frac{\pi s}{2}\right) \\
& \left(L i_{1-s}\left(-e^{-\frac{i a \pi}{b}}\right)+L i_{1-s}\left(-e^{\frac{i a \pi}{b}}\right)-L i_{1-s}\left(-e^{-\frac{i c \pi}{b}}\right)-L i_{1-s}\left(-e^{\frac{i c \pi}{b}}\right)\right)
\end{aligned}
$$

Proof. Use Equation (11) and take the first partial derivative with respect to $a$ and replace $s \rightarrow s-1$. Next form a second equation by replacing $a \rightarrow c$ and take their difference and simplify.

\subsection{Derivation of Entry 4.119 in Gradshteyn and Ryzhik}

Proposition 8.

$$
\int_{0}^{\infty} \frac{(1-\cosh (p x)) \operatorname{csch}(q x)}{x} d x=\log \left(\cos \left(\frac{\pi p}{2 q}\right)\right)
$$

Proof. Use Equation (14) and form a second equation by replacing $a \rightarrow c$ and taking their difference to get

$$
\begin{aligned}
& \int_{0}^{\infty} x^{s-1} \operatorname{csch}(b x)(\cosh (c x)-\cosh (a x)) d x=-\frac{1}{2} \pi^{s} b^{-s} \sec \left(\frac{\pi s}{2}\right) \\
& \left(\operatorname{Li}_{1-s}\left(-e^{-\frac{i a \pi}{b}}\right)+\operatorname{Li}_{1-s}\left(-e^{\frac{i a \pi}{b}}\right)-\operatorname{Li}_{1-s}\left(-e^{-\frac{i c \pi}{b}}\right)-\operatorname{Li}_{1-s}\left(-e^{\frac{i c \pi}{b}}\right)\right)
\end{aligned}
$$

Next set $c=s=0$ and simplify. Note that an analogous technique has also been used in the study of combinatorial geometry see [15].

\section{Proposition 9.}

$$
\int_{0}^{\infty} x^{2}(\sinh (p x)-1) \operatorname{csch}(q x) d x=\frac{\pi^{3} \tan \left(\frac{\pi p}{2 q}\right) \sec ^{2}\left(\frac{\pi p}{2 q}\right)-14 \zeta(3)}{4 q^{3}}
$$

Proof. Use Equation (14) and form a second equation by taking the first partial derivative with respect to $a$ then replace $s \rightarrow s-1$ and $a \rightarrow c$ and take their difference to get

$$
\begin{aligned}
& \int_{0}^{\infty} x^{s-1} \operatorname{csch}(b x)(\sinh (c x)-\cosh (a x)) d x \\
& =\frac{1}{2} \pi^{s} b^{-s} \\
& \quad-\sec \left(\frac{\pi s}{2}\right)\left(\operatorname{Li}_{1-s}\left(-e^{-\frac{i a \pi}{b}}\right)+\operatorname{Li}_{1-s}\left(-e^{\frac{i a \pi}{b}}\right)\right) \\
& \left.\quad-i \csc \left(\frac{\pi s}{2}\right)\left(\operatorname{Li}_{1-s}\left(-e^{-\frac{i c \pi}{b}}\right)-\operatorname{Li}_{1-s}\left(-e^{\frac{i c \pi}{b}}\right)\right)\right)
\end{aligned}
$$

Next, set $a=0$ and simplify using entry (1) in Table below (25:12:5) in [14] to get

$$
\begin{array}{r}
\int_{0}^{\infty} x^{s-1} \operatorname{csch}(b x)(\sinh (c x)-1) d x=\frac{1}{2} \pi^{s} b^{-s}\left(\left(1-2^{s}\right) \zeta(1-s) \sec \left(\frac{\pi s}{2}\right)\right. \\
\left.-i \csc \left(\frac{\pi s}{2}\right)\left(\operatorname{Li}_{1-s}\left(-e^{-\frac{i c \pi}{b}}\right)-\operatorname{Li}_{1-s}\left(-e^{\frac{i c \pi}{b}}\right)\right)\right)
\end{array}
$$

followed by applying L'Hopital's rule to the right-hand side as $s=3$ and set $c \rightarrow p$ and $b \rightarrow q$ and simplify to get the stated result.

\section{Proposition 10.}

$$
\int_{0}^{\infty} \operatorname{csch}(b x)(\sin (c x)-\sin (a x)) d x=\frac{\pi\left(\tanh \left(\frac{\pi c}{2 b}\right)-\tanh \left(\frac{\pi a}{2 b}\right)\right)}{2 b}
$$


Proof. Use Equation (14) and form a second equation by replacing $a \rightarrow c$ and take their difference to get

$$
\begin{aligned}
& \int_{0}^{\infty} x^{s-1} \operatorname{csch}(b x)(\sinh (c x)-\sinh (a x)) d x \\
& =\frac{1}{2} i \pi^{s} b^{-s} \csc \left(\frac{\pi s}{2}\right)\left(\operatorname{Li}_{1-s}\left(-e^{-\frac{i a \pi}{b}}\right)-\operatorname{Li}_{1-s}\left(-e^{\frac{i a \pi}{b}}\right)-\operatorname{Li}_{1-s}\left(-e^{-\frac{i c \pi}{b}}\right)\right. \\
& \left.+\operatorname{Li}_{1-s}\left(-e^{\frac{i c \pi}{b}}\right)\right)
\end{aligned}
$$

Next we set $s=1, a=a i, c=c i$ and simplify.

4.7. Derivation of Entry 4.121.2 in Gradshteyn and Ryzhik

Proposition 11.

$$
\int_{0}^{\infty} \frac{\operatorname{csch}(b x)(\cos (c x)-\cos (a x))}{x} d x=\log \left(\cosh \left(\frac{\pi a}{2 b}\right) \operatorname{sech}\left(\frac{\pi c}{2 b}\right)\right)
$$

Proof. Use Equation (14) and replace $s \rightarrow s-1$ and take the first partial derivative with respect to $a$ then replace $a \rightarrow c$ to form a second equation and take their difference to get

$$
\begin{aligned}
& \int_{0}^{\infty} x^{s-1} \operatorname{csch}(b x)(\cosh (c x)-\cosh (a x)) d x \\
& =-\frac{1}{2} \pi^{s} b^{-s} \sec \left(\frac{\pi s}{2}\right)\left(\operatorname{Li}_{1-s}\left(-e^{-\frac{i a \pi}{b}}\right)+\operatorname{Li}_{1-s}\left(-e^{\frac{i a \pi}{b}}\right)-\operatorname{Li}_{1-s}\left(-e^{-\frac{i c \pi}{b}}\right)\right. \\
& \left.-\mathrm{Li}_{1-s}\left(-e^{\frac{i c \pi}{b}}\right)\right)
\end{aligned}
$$

Next we set $s=0, a=a i, c=c i$ and simplify.

\section{Proposition 12.}

$$
\begin{aligned}
& \int_{0}^{\infty} x \operatorname{csch}(b x)(\sinh (c x)-\cosh (a x)) d x \\
& =-\frac{\pi}{b^{2}}\left(\frac{\pi}{2 \cos \left(\frac{\pi a}{b}\right)+2}+i\left(L i_{-1}^{\prime}\left(-e^{-\frac{i \pi c}{b}}\right)-L i_{-1}^{\prime}\left(-e^{\frac{i \pi c}{b}}\right)\right)\right)
\end{aligned}
$$

Proof. Use Equation (14) and form a second equation by replacing $a \rightarrow c$ and taking their difference to get

$$
\begin{aligned}
& \int_{0}^{\infty} x^{s-1} \operatorname{csch}(b x)(\sinh (c x)-\cosh (a x)) d x \\
& \begin{aligned}
=\frac{1}{2} \pi^{s} b^{-s}( & -\sec \left(\frac{\pi s}{2}\right)\left(\operatorname{Li}_{1-s}\left(-e^{-\frac{i a \pi}{b}}\right)+\operatorname{Li}_{1-s}\left(-e^{\frac{i a \pi}{b}}\right)\right) \\
& \left.\quad-i \csc \left(\frac{\pi s}{2}\right)\left(\operatorname{Li}_{1-s}\left(-e^{-\frac{i c \pi}{b}}\right)-\operatorname{Li}_{1-s}\left(-e^{\frac{i c \pi}{b}}\right)\right)\right)
\end{aligned}
\end{aligned}
$$

Next we apply L'Hopital's theorem to the right-hand side as $s \rightarrow 2$ and simplify.

\subsection{Derivation of New Entries for Table 4.122 in Gradshteyn and Ryzhik}

\section{Proposition 13.}

$$
\begin{aligned}
& \int_{0}^{\infty} x^{s-1} \operatorname{csch}\left(\frac{x}{2}\right) \sinh (m x) \sinh (p x) d x \\
& =2^{s-2} \pi^{s} \sec \left(\frac{\pi s}{2}\right)\left(-L i_{1-s}\left(-e^{-2 i(m-p) \pi}\right)-L i_{1-s}\left(-e^{2 i(m-p) \pi}\right)\right. \\
& \left.+L i_{1-s}\left(-e^{-2 i(m+p) \pi}\right)+L i_{1-s}\left(-e^{2 i(m+p) \pi}\right)\right)
\end{aligned}
$$


Proof. Use Equation (8) and replace $m \rightarrow m+p$ to form a second equation and take their difference to get

$$
\begin{gathered}
\int_{0}^{\infty} \operatorname{csch}\left(\frac{x}{2}\right) \sinh (m x)\left(e^{p x}(\log (a)-i x)^{k}+e^{-p x}(\log (a)+i x)^{k}\right) d x \\
=i(2 \pi)^{k+1} e^{-2 i \pi(m-p)}\left(\Phi\left(-e^{-2 i(m-p) \pi},-k, \frac{\log (a)}{2 \pi}+1\right)\right. \\
\left.-e^{4 i \pi m} \Phi\left(-e^{2 i(m+p) \pi},-k, \frac{\log (a)}{2 \pi}+1\right)\right)
\end{gathered}
$$

Next we form a third equation by setting $p \rightarrow-p$ and taking the difference from the second equation to get

$$
\begin{gathered}
\int_{0}^{\infty} \operatorname{csch}\left(\frac{x}{2}\right) \sinh (m x)\left(e^{p x}(\log (a)-i x)^{k}+e^{-p x}(\log (a)+i x)^{k}\right) d x \\
=i(2 \pi)^{k+1} e^{-2 i \pi(m-p)}\left(\Phi\left(-e^{-2 i(m-p) \pi},-k, \frac{\log (a)}{2 \pi}+1\right)\right. \\
\left.-e^{4 i \pi m} \Phi\left(-e^{2 i(m+p) \pi},-k, \frac{\log (a)}{2 \pi}+1\right)\right)
\end{gathered}
$$

Next we set $a=1$ and simplify using Equation (64:12:2) in [14] to get the stated result.

\subsection{Derivation of New Entry 4.122.3 in Gradshteyn and Ryzhik}

Proposition 14.

$$
\int_{0}^{\infty} \frac{\operatorname{csch}\left(\frac{x}{2}\right) \sinh (m x) \sinh (p x)}{x} d x=\frac{1}{2} \log (\cos (\pi(m-p)) \sec (\pi(m+p)))
$$

Proof. Use (31) and set $s=0$ and simplify.

4.10. Derivation of New Entry 4.122.4 in Gradshteyn and Ryzhik

\section{Proposition 15.}

$$
\int_{0}^{\infty} \operatorname{csch}\left(\frac{x}{2}\right) \cosh (m x) \sinh (p x) d x=\frac{1}{2} \pi(\tan (\pi(m+p))-\tan (\pi(m-p)))
$$

Proof. Use Equation (34) and take the first partial derivative with respect to $m$ and simplify.

\section{Discussion}

In this paper, we have presented a novel method for deriving some interesting definite integrals using contour integration. We will be extending our study of these types of integrals using our method and expanding the Section 1 Table 1 . The results presented were numerically verified for both real and imaginary and complex values of the parameters in the integrals using Mathematica by Wolfram.

Author Contributions: Conceptualization, R.R., funding acquisition, A.S., supervision, A.S. Both authors have read and agreed to the published version of the manuscript.

Funding: The Natural Sciences and Engineering Research Council of Canada, Grant number, 504070.

Institutional Review Board Statement: Not applicable.

Informed Consent Statement: Not applicable. 
Data Availability Statement: Not applicable.

Conflicts of Interest: The authors declare no conflict of interest.

\section{References}

1. Berndt, B.C. Ramanujan's Notebooks Part 2; Springer: New York, NY, USA, 1989.

2. Mohr, C.B.O. Theory of heavy-ion elastic collisions revisited. Aust. J. Phys. 1984, 37, 9-16. [CrossRef]

3. Dalmao, F.; Estrade, A.; León, J.R. On 3-dimensional Berry's model. ALEA Lat. Am. J. Probab. Math. Stat. 2021, 18, 379-399. [CrossRef]

4. Hagentoft, C.-E. Heat losses and temperature in the ground under a building with and without ground water flow-II. Finite ground water flow rate. Build. Environ. 1996, 31, 13-19. [CrossRef]

5. Hans, S. Advanced Calculus: Of Real-Valued Functions of a Real Variable and Vector-Valued Functions of a Vector Variable, 1st ed.; Houghton Mifflin Co.: Boston, MA, USA, 1 January 1974; pp. 1-671.

6. Abro, K.A.; Gomez-Aguilar, J.F. Role of Fourier sine transform on the dynamical model of tensioned carbon nanotubes with fractional operator. Math. Methods Appl. Sci. 2020. [CrossRef]

7. Agarwal, R.P.; Bazighifan, O.; Ragusa, M.A. Nonlinear neutral delay differential equations of fourth-order: Oscillation of solutions . Entropy 2021, 23, 129. [CrossRef] [PubMed]

8. Reynolds, R.; Stauffer, A. A Method for Evaluating Definite Integrals in Terms of Special Functions with Examples. Int. Math. Forum 2020, 15, 235-244. [CrossRef]

9. Gradshteyn, I.S.; Ryzhik, I.M. Tables of Integrals, Series and Products, 6th ed.; Academic Press: Cambridge, MA, USA, 2000.

10. Brychkov, Y.A.; Marichev, O.I.; Savischenko, N.V. Handbook of Mellin Transforms, 1st ed.; Chapman and Hall/CRC: Boca Raton, FL, USA, 2018.

11. Olver, F.W.J.; Lozier, D.W.; Boisvert, R.F.; Clark, C.W. (Eds.) NIST Digital Library of Mathematical Functions; With 1 CD-ROM (Windows, Macintosh and UNIX). MR 2723248 (2012a:33001); U.S. Department of Commerce, National Institute of Standards and Technology: Washington, DC, USA; Cambridge University Press: Cambridge, UK, 2010.

12. Erdéyli, A.; Magnus, W.; Oberhettinger, F.; Tricomi, F.G. Higher Transcendental Functions; McGraw-Hill Book Company, Inc.: New York, NY, USA; Toronto, ON, Canada; London, UK, 1953; Volume I.

13. Abramowitz, M.; Stegun, I.A. (Eds.) Handbook of Mathematical Functions with Formulas, Graphs, and Mathematical Tables, 9th ed.; Dover: New York, NY, USA, 1982.

14. Oldham, K.B.; Myland, J.C.; Spanier, J. An Atlas of Functions: With Equator, the Atlas Function Calculator, 2nd ed.; Springer: New York, NY, USA, 2009.

15. Shang, Y. On the Degree Sequence of Random Geometric Digraphs. Appl. Math. Sci. 2010, 4, $2001-2012$. 